DCPT-05/39

\title{
Winding tachyons in asymptotically supersymmetric black strings
}

\author{
Simon F. Ross* \\ Centre for Particle Theory, Department of Mathematical Sciences \\ University of Durham, South Road, Durham DH1 3LE, U.K.
}

\begin{abstract}
We show that in the D1-D5 system with angular momentum, there can be localised tachyonic winding string modes in the interior of the spacetime even if we choose a spin structure which preserves supersymmetry in the asymptotic region. We consider cases where the tachyonic region extends outside the event horizon, and argue that the natural endstate of tachyon condensation in almost all cases is one of the solitonic solutions which correspond to special microstates of the D1-D5 system.
\end{abstract}

\section{Introduction and Summary}

The condensation of localised closed string tachyons has been found to have a rich physics. $^{1}$ In the pioneering work of [3], it was argued that the condensation of a twisted sector tachyon could cause an orbifold singularity to decay into flat space. This was generalised to twisted circles in [4, and an interesting discussion of the null orbifold was recently given in [5]. The condensation of a winding tachyon in a spacetime with a circle with antiperiodic fermions has been argued to lead to the pinching off of cycles, changing the spatial topology [6], and to the decay of a black string into a static or expanding bubble [7]. It has been conjectured that such winding string tachyon condensation can provide a chronology protection mechanism [8]. The condensation of a winding tachyon on a time-dependent circle has even been argued to provide a perturbative resolution of big-bang like singularities [9].

The aim of this paper is to provide a further interesting example of tachyon condensation, modifying the example discussed in [7] so that the spacetime is asymptotically supersymmetric, so that the only instability is the localised winding tachyon.

In [7, a black string wrapped on a circle with antiperiodic boundary conditions was considered. The circle will shrink near the string, so for a suitable choice of parameters there is a winding mode which becomes tachyonic outside the event horizon. It was

*S.F.Ross@durham.ac.uk

${ }^{1}$ Useful reviews of these developments, with more extensive references, are [1, 2]. 
argued that the condensation of this tachyon will cause the black string to decay into a charged bubble solution with no horizon.

This example provides a new insight into the endpoint of Hawking evaporation for such black strings. However, the antiperiodic boundary conditions give a non-zero Casimir energy density in the asymptotic region. This will cause the circle to contract, and its back-reaction could spoil the asymptotic flatness of the solution. Also, flat space with this supersymmetry-breaking compactification has a non-perturbative instability to decay to the Witten 'bubble of nothing' 10. Although the tachyon condensation is more dynamically efficient than this non-perturbative decay, this does show that the instability of the black string solution is not truly localised. In [7], it was suggested that these issues could be addressed by taking the circle to be a cycle in a Riemann surface of the type considered in [11, where fluxes and branes would stabilise the circle. They nonetheless imply it is worth seeking an example of a black string solution with a similar winding string tachyon, but where the circle has periodic boundary conditions in the asymptotic region, so that supersymmetry will be restored asymptotically, and the tachyon provides the only instability.

This sounds like an impossible request, since to have a tachyon in the winding sector, the fermions have to be antiperiodic around a circle. However, as we will see below, it can actually be easily achieved by exploiting the idea underlying the smooth solitons found in 12, 13, 14, 15, 16, 17]: in the D1-D5 system with angular momentum, in the asymptotically supersymmetric sector, we can twist the asymptotic $S^{1}$ by a rotation in the $S^{3}$ to obtain a circle with antiperiodic fermions in the near-horizon region. Such a circle could then have a tachyon in a string winding sector.

We develop this example in the next section, and discuss its near-horizon limit in section 3. We will see that as in the case without angular momentum, there are choices of parameters in the D1-D5 system for which a tachyon appears outside the event horizon, and the curvatures and string coupling are small at the horizon. Physically what is happening is that the interaction between the charges and the angular momentum can be tuned so that the black string causes some twisted circle to shrink. The near-horizon limit of these solutions is found to be identical to the case with zero angular momentum, clearly showing that the local analysis of the tachyon near the horizon for these examples will be exactly the same despite the change in asymptotic spin structure.

It is important to note that the appearance of a tachyon on a twisted circle is very special to the example we have considered. It is not simply a matter of adding angular momentum; the twisted circle has small size near the horizon as a result of the presence of a $d y d \phi$ cross term in the metric, and such cross terms appear to be special to the D1-D5 system. ${ }^{2}$ The cross term disappears if we set one of the charges to zero, and no such term appears in the metric for a rotating $p$-brane with a single charge [21, 22, 23]. The presence of the cross term is also (obviously) not dualityinvariant, although the tachyon should appear in some other way in related duality

\footnotetext{
${ }^{2}$ The other special feature of the D1-D5 system is that this is the only case with a regular supersymmetric rotating black hole solution [18] (see [19, 20] for further discussion of this fact). One wonders if these facts are in any way related.
} 
frames (see discussion below).

In section 4, we consider the endpoint of this tachyon condensation. Here we find a major difference relative to the previous case: In [7, there was a static bubble only for certain values of the parameters, and the black string otherwise decayed into an expanding bubble which eventually consumed the entire spacetime. By contrast, we find that there is a smooth stationary soliton solution from [17] for all values of the parameters. This provides a natural endpoint for the decay of the tachyon in almost all cases. The only exception is the case of unit twist, where the soliton is BPS, and turns out to be larger than the tachyonic black string if the charge is large, $Q / R^{2} \gg 1$. It is not clear what happens in this case: the decay may eventually settle down to the soliton after some non-trivial evolution, or there may some other endstate for the decay, although no such solution is explicitly identified. We argue that expanding bubbles do not appear in the decay of these black strings. It would be valuable to have a deeper understanding of the connection between this result and the choice of spin structure.

In addition to providing a new example of localised closed string tachyon condensation, this analysis gives an intriguing new relation between the black string solutions and the smooth solitons. Mathur and collaborators have argued that these solitons provide a geometrical description of the microstates responsible for the black string's entropy, and that the black string only emerges as a 'coarse-grained' description of the microstate geometries [24, 25] (see [26] for a review). It is therefore quite interesting that this tachyon condensation provides a stringy mechanism by which a 'naïve' black string geometry can decay into one of the smooth solitons. However, there are plenty of black strings which do not have such an instability: our argument only applies to the special class of solutions where a circle is smaller than the string scale outside of the horizon. Thus, while this observation is suggestive, its relation to the Mathur programme is not entirely clear.

In [7], it was argued that any black string will eventually decay by Hawking radiation until it reaches the point where this instability sets in. The decay to a soliton does not seem to have the same inevitability in our case, since the black strings which decay via the tachyon condensation have a non-zero angular momentum, and we would expect that any initial angular momentum on a macroscopic black string will quickly be lost through preferential Hawking radiation of co-rotating modes (although a detailed analysis of this has not been carried out for these higher-dimensional black holes). That is, a generic black string will shrink by Hawking radiation, but it may not reach a regime where a circle with antiperiodic boundary conditions is string scale outside the horizon before the curvatures reach the string scale, and corrections to the classical geometry become important.

Finally, we can study this system when the asymptotic circle is smaller than the string scale, which will make a prediction for the behaviour of the T-dual spacetime. The periodic spin structure implies the spacetime is still stable asymptotically, and our analysis still predicts a winding mode tachyon in the region near the horizon for appropriate choices of the parameters. In the T-dual system, this winding string gets mapped to a string with momentum along a dual circle, wrapped on an $S^{1}$ in the $S^{3}$. Although this is a contractible circle, which does not correspond to a conserved 
winding number, wrapping this circle may play a role in making this state tachyonic, via a coupling similar to that of long strings [27]. A project for future investigation is to see if we can identify and understand this localised tachyon in the dual system. ${ }^{3}$ The simplest case to consider is probably the NS5-P system. We would expect that the soliton solutions would still be appropriate endpoints of tachyon condensation, even though they are not smooth spacetimes in the T-dual variables. They would be interpreted as geometries with explicit brane sources.

\section{Black strings with periodic boundary conditions}

The string-frame metric for the D1-D5 solution with one angular momentum is [29, 30. (in a form previously employed in [17])

$$
\begin{aligned}
\mathrm{d} s^{2}= & \frac{1}{\sqrt{\tilde{H}_{1} \tilde{H}_{5}}}\left[-(f-M)\left(\mathrm{d} t-(f-M)^{-1} M \cosh \delta_{1} \cosh \delta_{5} a_{1} \cos ^{2} \theta \mathrm{d} \psi\right)^{2}\right. \\
& \left.+f\left(\mathrm{~d} y+f^{-1} M \sinh \delta_{1} \sinh \delta_{5} a_{1} \sin ^{2} \theta \mathrm{d} \phi\right)^{2}\right]+\sqrt{\frac{\tilde{H}_{1}}{\tilde{H}_{5}}} \sum_{i=1}^{4} \mathrm{~d} z_{i}^{2} \\
& +\sqrt{\tilde{H}_{1} \tilde{H}_{5}}\left(\frac{\mathrm{d} r^{2}}{r^{2}+a_{1}^{2}-M}+\mathrm{d} \theta^{2}+\frac{r^{2} \sin ^{2} \theta}{f} \mathrm{~d} \phi^{2}+\frac{\left(r^{2}+a_{1}^{2}-M\right) \cos ^{2} \theta}{f-M} \mathrm{~d} \psi^{2}\right) .
\end{aligned}
$$

We are focusing on the simplest case where the twisting phenomenon occurs, two charges and a single angular momentum. It is useful to focus on the case with no momentum along the $y$ circle, as it makes it clear such a momentum is not playing a role in this story, but the discussion could easily be generalised to include momentum charge and two angular momenta. The dilaton field is $e^{2 \Phi}=\tilde{H}_{1} / \tilde{H}_{5}$. See [15] for the value of the $\mathrm{RR}$ field, which we will not need. The functions appearing in the above metric are

$$
\tilde{H}_{i}=f+M \sinh ^{2} \delta_{i}, \quad f=r^{2}+a_{1}^{2} \sin ^{2} \theta
$$

The $z_{i}$ are coordinates on a $T^{4}$ of volume $V$, and we take $y$ to be a periodic coordinate on an $S^{1}, y \sim y+2 \pi R$. In units where $G^{(5)}=G^{(10)} /(2 \pi R V)=\pi / 4$, the mass, angular momentum and charges are

$$
\begin{gathered}
M_{A D M}=\frac{M}{2}\left(\cosh 2 \delta_{1}+\cosh 2 \delta_{5}+1\right), \\
J_{\psi}=-M a_{1} \cosh \delta_{1} \cosh \delta_{5} \\
Q_{1}=M \sinh \delta_{1} \cosh \delta_{1} \\
Q_{5}=M \sinh \delta_{5} \cosh \delta_{5}
\end{gathered}
$$

\footnotetext{
${ }^{3}$ An effective action with a field which becomes tachyonic near the event horizon of a charged black hole was recently discussed in [28. This might be a useful model for the T-dual physics.
} 
If $Q_{1}=Q_{5}$, the dilaton is constant, and the S-dual of this solution is an F1-NS5 system with the same metric (2.1). This is then the generalisation of the two-charge black string considered in [7] to include angular momentum.

This solution has a horizon at $r_{+}^{2}=M-a_{1}^{2}$. Assume $M>a_{1}^{2}$, so that we are discussing a black string, with a regular event horizon. The $y$ circle is then not contractible in the interior of the spacetime, so we are free to choose the spin structure on this circle. We will take periodic boundary conditions for the fermions on the $y$ circle, so that in the asymptotic region $r \rightarrow \infty$, the solution approaches $\mathbb{R}^{5,1} \times T^{4} \times S^{1}$ with unbroken supersymmetry.

We then need to identify a suitable circle with antiperiodic fermions. The Killing vector

$$
\xi=\partial_{y}-\frac{m}{R} \partial_{\phi}
$$

has closed orbits for integer $m$. If the integer $m$ is related to the parameters of the solution by

$$
m=\frac{R a_{1}}{M \sinh \delta_{1} \sinh \delta_{5}},
$$

the proper size of this $S^{1}$ vanishes as $r^{2} \rightarrow 0$. Thus, this circle shrinks to zero size in the interior of the spacetime. If $m$ is odd, the fermions are antiperiodic on this circle, and we expect that the spectrum of winding modes around this circle includes a tachyon. ${ }^{4}$ In the case without angular momentum, we know that the tension of the wrapped black string will make the asymptotic circle shrink near the string. Here, the combination of this effect with the $d y d \phi$ cross term in the metric causes a different, twisted circle to shrink when we tune the angular momentum as in (2.8). Note that the asymptotic circle remains of finite size at $r^{2}=0$ for any nonzero $a_{1}$, even though we have not introduced any momentum along this direction. ${ }^{5}$

For $M>a_{1}^{2}, r=0$ is a spacelike surface, so this is similar to the end of the universe tachyon studied in [9]. It would be interesting to further explore the time-dependent physics of the tachyon condensation behind the horizon of the black string. In this paper, however, we content ourselves with studying the generalisation of [7], the case where the region in which a winding mode is tachyonic extends outside the event horizon. The proper size of this twisted circle at the horizon is given by

$$
\|\xi\|^{2} R^{2}=\frac{r_{+}^{2} R^{2}}{\sqrt{\tilde{H}_{1} \tilde{H}_{5}}}\left[1+\frac{a_{1}^{2} \sin ^{2} \theta}{M^{2} \sinh ^{2} \delta_{1} \sinh ^{2} \delta_{5}}\left(f+M \sinh ^{2} \delta_{1}+M \sinh ^{2} \delta_{5}\right)\right] .
$$

Thus, for sufficiently small values of $r_{+}^{2}$, the proper size of the orbits of $\xi$ is smaller than the string scale outside the event horizon.

These geometries thus provide examples where we expect tachyon condensation to change the geometry outside the event horizon, and the geometry at large distances is

\footnotetext{
${ }^{4}$ In fact, such a tachyon should exist for some open range of parameters around the values implied by (2.8), for which this circle becomes smaller than the string scale for $r^{2}$ near zero. We focus however on the specific choice of parameters in (2.8), as this will allow us to make more definite statements.

${ }^{5}$ The $y$ circle does go to zero size for $r^{2}=0, \theta=0$, but this is special to the case with one angular momentum. In the general case, it would remain of finite size for all $\theta$.
} 
supersymmetry-preserving, so the instability is, at least initially, a genuinely localised phenomenon.

\section{Near-horizon limit}

In the previous section, we argued heuristically that there should be a winding string tachyon where the twisted circle is small enough. However, there is only one conserved winding number in the spacetime, not one for every choice of $m$ : the circle we are considering can be smoothly deformed into the $y$ circle (or any other such twisted circle) at constant $r$. One might therefore worry that a careful analysis of the spectrum might find that the supersymmetry on the $y$ circle forbids the appearance of tachyons. In this section, we consider the near-horizon limit of (2.1), where it is easier to work out the spectrum of string states, and can see that there really is a tachyon. This will also allow us to relate this case to the case studied in [7].

If we consider near-extremal black strings with the radius $R$ large compared to the other scales in the geometry, $R \gg \sqrt{Q_{1}}, \sqrt{Q_{5}}, \sqrt{M}$, the geometry (2.1) has a near horizon limit which is locally $\mathrm{AdS}_{3} \times S^{3}$ with radii $\ell_{A d S}=\ell_{S}=\ell=\left(Q_{1} Q_{5}\right)^{1 / 4}$. For the black string solution, the near-horizon geometry is a BTZ black hole $\times S^{3}[31$,

$$
\begin{aligned}
\mathrm{d} s^{2}= & -\left(\frac{\rho^{2}}{\ell^{2}}-M_{3}\right) d \tau^{2}+\left(\frac{\rho^{2}}{\ell^{2}}-M_{3}\right)^{-1} d \rho^{2}+\rho^{2} d \varphi^{2} \\
& +\ell^{2}\left[d \theta^{2}+\sin ^{2} \theta(d \phi+m d \varphi)^{2}+\cos ^{2} \theta\left(d \psi+\frac{m}{\ell} d \tau\right)^{2}\right]
\end{aligned}
$$

with

$$
M_{3}=\frac{R^{2}}{\ell^{4}}\left(M-a_{1}^{2}\right),
$$

where we have defined the new coordinates

$$
\varphi=\frac{y}{R}, \quad \tau=\frac{t \ell}{R} \quad \rho^{2}=\frac{R^{2}}{\ell^{2}} r^{2} .
$$

By assumption, the fermions are periodic around the $\varphi$ circle. However, if we make the large coordinate transformation $\tilde{\phi}=\phi+m \varphi, \tilde{\psi}=\psi+m \tau / \ell$ to bring the metric to a direct product form, the fermions will be antiperiodic around $\varphi$ at fixed $\tilde{\phi}, \tilde{\psi}$ if $m$ is odd. This solution then agrees exactly with the near-horizon limit of the two-charged black strings without angular momentum considered in [7]. This direct product geometry is just a quotient $\mathrm{AdS}_{3} / \mathbb{Z} \times S^{3}$, and using the methods of [32, 33] one can explicitly check that there is a tachyon in the twisted sector of this orbifold when the circle becomes smaller than the string scale. This near-horizon limit also shows that the curvatures and string coupling can be kept small at the horizon, so that corrections to this perturbative worldsheet analysis are under control. ${ }^{6}$

This system clearly deserves further study. Considering the geometry with NS fluxes, it ought to be possible to study this tachyon from the worldsheet point of

\footnotetext{
${ }^{6}$ Also, the radial proper distance over which the circle remains small is of order the AdS scale $\left(Q_{1} Q_{5}\right)^{1 / 4}$, so the circle's size is varying slowly for a large AdS space.
} 
view, following [3, 6, 5]. For now, the lesson to draw from this is that in the nearhorizon region, the tachyon story is the same, independent of whether the compact circle direction in the full asymptotically flat solution has periodic or anti-periodic fermions. Thus, we believe there is a winding string tachyon in the full asymptotically flat solution considered in the previous section.

\section{Endpoint of tachyon condensation}

Having argued that there is a tachyon for some black string solutions of the form (2.1), we should now investigate what the endstate of condensation of this tachyon is.

In the near-horizon limit discussed in the previous section, the natural candidate for the endpoint is global $\mathrm{AdS}_{3} \times S^{3}$. This immediately suggests that for the nearextremal black strings which have this near-horizon limit, the natural endpoint for the decay of the tachyon will be the soliton of [17] which embeds global $\mathrm{AdS}_{3} \times S^{3}$ in an asymptotically flat spacetime, with the $S^{1}$ direction in $\mathrm{AdS}_{3}$ corresponding to the circle labelled by the same Killing vector $\xi=\partial_{y}-\frac{m}{R} \partial_{\phi} \cdot{ }^{7}$ But what about more general black strings: do they also decay into smooth solitons?

Here there is a significant difference between the present discussion and [7]. It was observed in [7] that the static bubble solution only existed for a range of values of the parameters $Q, R$. In our case, however, it was found in [17] that there is a soliton solution for any choice of $Q_{1}, Q_{5}$, and $R$ for each positive integer $m$. If we consider for simplicity $Q_{1}=Q_{5}=Q$, we can illustrate this explicitly: the radius of the circle for a smooth soliton is fixed by

$$
R=\frac{\tilde{M} \sinh ^{2} \tilde{\delta}}{\sqrt{\tilde{a}_{1}^{2}-\tilde{M}}}
$$

where $\tilde{M}, \tilde{a}_{1}$ are the parameters for the smooth soliton, which need not agree with the parameters of the original black string solution. Together with (2.8), this implies

$$
\tilde{M}=\frac{\tilde{a}_{1}^{2}}{m^{2}}\left(m^{2}-1\right)
$$

The conditions (2.84.1) and the expression for the charge, $Q=\tilde{M} \sinh \tilde{\delta} \cosh \tilde{\delta}$, fix the parameters $\tilde{M}, \tilde{a}_{1}$ and $\tilde{\delta}$. We use (2.8) and (4.1) to write $\tilde{a}_{1}$ and $\tilde{M}$ in terms of $\tilde{\delta}$. The expression for the charge then yields an equation for $\tilde{\delta}$,

$$
\frac{Q}{R^{2}}\left(m^{2}-1\right) \tanh ^{3} \tilde{\delta}+\tanh ^{2} \tilde{\delta}-1=0
$$

For $m=1$, the solution is $\tanh \tilde{\delta}=1$. For $m \neq 1$, we can rewrite this as an expression for $Q / R^{2}$ in terms of $\tilde{\delta}$ :

$$
\frac{Q}{R^{2}}=\frac{\cosh \tilde{\delta}}{\left(m^{2}-1\right) \sinh ^{3} \tilde{\delta}} .
$$

\footnotetext{
${ }^{7}$ Note if the curvature of the metric near the horizon is small, we can only make the circle associated with one choice of $m$ small.
} 
The RHS is unbounded, so in contrast to [7, a smooth soliton solution exists for any $R, Q$. (Note also that this is a monotonic function of $\tilde{\delta}$, so there is a single soliton solution for each $Q, R$.) Thus, we can conjecture that whenever the asymptotically supersymmetric black string solution (2.1) has a winding string tachyon, the endpoint of tachyon condensation is a smooth soliton solution.

If the black string solution is going to decay into the soliton solution, it must also have a larger mass. In the near-horizon limit, the BTZ black hole clearly has larger energy than global AdS, so this condition is satisfied. As we now see, this remains true for the asymptotically flat solution, at least when it satisfies (2.8).

The condition (2.8) and the condition that we fix the charge leave us one free parameter in the black string, but it can only vary in a small range: the black string solution only has a tachyon outside the horizon if $M-a_{1}^{2}$ is small (and positive). The minimum value of the mass for fixed charge is attained when we have the smallest possible value for $M$, so we will take

$$
M=a_{1}^{2}
$$

in considering the black string. We can then solve for the parameters in terms of the charges, which gives a very similar expression for $Q / R^{2}$,

$$
\frac{Q}{R^{2}} m^{2} \tanh ^{3} \delta+\tanh ^{2} \delta-1=0,
$$

or

$$
\frac{Q}{R^{2}}=\frac{\cosh \delta}{m^{2} \sinh ^{3} \delta} .
$$

The small change in the denominator on the RHS implies that that for a given value of $Q / R^{2}, \delta<\tilde{\delta}$ (also true for $m=1$, as $\tilde{\delta}=\infty$ ) so for fixed charge $M>\tilde{M}$, and hence the ADM mass of the black string is always larger than the ADM mass of the soliton with the same values of $Q, R$ and $m$.

In 7], another condition was imposed: the size of the sphere at the static bubble was required to agree with the size of the sphere at the horizon in the original black string. This is a reasonable condition, corresponding to the intuition that the tachyon condensation should be happening locally near the horizon. Imposing it would seem likely to cause problems, however, since the soliton geometry is fixed by the choice of $Q$ and $R$, so we have no free parameters left to tune. Surprisingly, we will see that this condition is also satisfied in almost all cases.

As a warm-up, we discuss the two charge case in [7] in a way that makes it easy to compare to our case. The black string is parametrised by two parameters $r_{0}, \alpha$, in terms of which the charge is

$$
Q=r_{0}^{2} \sinh 2 \alpha .
$$

To have a tachyon outside the horizon, we choose the asymptotic circle periodicity to be

$$
R=l_{s} \cosh \alpha,
$$

which implies $\alpha \gg 1$ so that $R \gg l_{s}$. The static bubble solution obtained by analytic continuation has parameters $\tilde{r}_{0}, \tilde{\alpha}$, and is smooth if

$$
R=2 \pi \tilde{r}_{0} \cosh ^{2} \tilde{\alpha} .
$$


The charge $Q$ is given by the same expression (4.8), so $\tilde{\alpha}$ is determined by

$$
\frac{Q}{R^{2}}=\frac{\sinh \tilde{\alpha}}{\pi \cosh ^{3} \tilde{\alpha}}
$$

(compare (4.4) ). This has a maximum as a function of $\tilde{\alpha}$ at $\tilde{\alpha} \approx 1$. Thus, for $Q / R^{2} \lesssim 1$, there are two soliton solutions. We will be interested in the one with $\tilde{\alpha} \geq 1$. The charge is the same, so the ratio of proper sizes of the sphere at the horizon or bubble is

$$
\frac{r_{0}^{2} \cosh ^{2} \alpha}{\tilde{r}_{0}^{2} \cosh ^{2} \tilde{\alpha}}=\frac{\tanh \tilde{\alpha}}{\tanh \alpha}
$$

Now $\alpha \gg 1$, and for $Q / R^{2} \lesssim 1$, we can solve (4.11) for $\tilde{\alpha} \geq 1$. So for $Q / R^{2} \lesssim 1$, there is a static bubble which is roughly the same size as the black string. ${ }^{8}$ For $Q / R^{2}>1$, there is no static bubble of any size, and the black string was argued to decay to an expanding bubble.

Now let us return to the present case. Since our black string geometry is not spherically symmetric, it is technically more difficult to impose the condition that the geometry of the $S^{3}$ at the event horizon agrees with the geometry of the $S^{3}$ where the circle degenerates in the bubble. We will therefore content ourselves with a rough estimate of the size. Of course, when the black string has an $\mathrm{AdS}_{3} \times S^{3}$ near-horizon limit, this condition is trivially satisfied, as the $S^{3}$ in the near-horizon region is a round $S^{3}$ with a radius determined by the charges. In the more general case, we take $\sqrt{\tilde{H}_{1} \tilde{H}_{5}}\left(r_{+}\right)$as a rough estimate of the size of the sphere at the horizon. for equal charges, this is

$$
A \equiv \tilde{H}_{1}\left(r_{+}\right)=r_{+}^{2}+a_{1}^{2} \sin ^{2} \theta+M \sinh ^{2} \delta .
$$

Assuming $R \gg l_{s}$, (2.9) implies $r_{+}^{2}$ will be small compared to $\sqrt{\tilde{H}_{1} \tilde{H}_{5}}$, and hence the first term is negligible. Using (2.8), $a_{1}=m M \sinh ^{2} \delta / R$, so

$$
A=m^{2} \frac{M^{2}}{R^{2}} \sinh ^{4} \delta \sin ^{2} \theta+M \sinh ^{2} \delta=Q\left(m^{2} \frac{Q}{R^{2}} \tanh ^{2} \delta \sin ^{2} \theta+\tanh \delta\right) .
$$

We can make a similar estimate for the size of the minimum-area surface $r=0$ in the soliton,

$$
\tilde{A} \equiv \tilde{a}_{1}^{2} \sin ^{2} \theta+\tilde{M} \sinh ^{2} \tilde{\delta}=Q\left(m^{2} \frac{Q}{R^{2}} \tanh ^{2} \tilde{\delta} \sin ^{2} \theta+\tanh \tilde{\delta}\right) .
$$

There are then two cases: For $Q / R^{2} \lesssim 1$, (4.3) and (4.6) will give $\tanh \tilde{\delta} \sim 1$, $\tanh \delta \sim 1$, and consequently $\tilde{A} \sim A$ : the soliton is roughly the same size as the black string. This includes the case $Q / R^{2} \ll 1$, where the solution has an $\mathrm{AdS}_{3} \times S^{3}$ near-horizon limit.

\footnotetext{
${ }^{8}$ In [7], this result was obtained by considering general initial data, and finding that when $Q / R^{2} \lesssim$ 1 , the bubble which was the same size as the black string horizon was a local minimum of the mass, and hence corresponded to a static bubble.
} 
For $Q / R^{2} \gg 1$, (4.6) will give

$$
\tanh \delta \approx\left(\frac{R^{2}}{Q m^{2}}\right)^{1 / 3} \ll 1,
$$

So

$$
A \sim Q\left(\frac{Q}{R^{2}}\right)^{1 / 3} .
$$

For the soliton, there is now an important distinction between $m=1$ and $m \neq 1$. For $m=1, \tanh \tilde{\delta}=1$, and the soliton has $\tilde{A} \sim Q^{2} / R^{2}$, so

$$
\frac{\tilde{A}}{A} \sim\left(\frac{Q}{R^{2}}\right)^{2 / 3} \gg 1
$$

For $m \neq 1,(4.3)$ gives

$$
\tanh \tilde{\delta} \approx\left(\frac{R^{2}}{Q\left(m^{2}-1\right)}\right)^{1 / 3} \ll 1
$$

SO

$$
\tilde{A} \sim Q\left(\frac{Q}{R^{2}}\right)^{1 / 3}
$$

and $\tilde{A} \sim A$.

Thus, in almost all cases, the rough size of the minimum area surface in the soliton and the horizon sphere in the black string agree. This works even though both the black string and the soliton are far from BPS in some cases. ${ }^{9}$ The smooth soliton solution then provides a natural endstate for tachyon condensation in the black string. The only exception is the case $m=1, Q / R^{2} \gg 1$, where the minimum radius in the soliton is much bigger than the black string's horizon radius. It is not clear what happens in this case. The soliton is still physically available as a possible endstate for the tachyon decay in this case, but if the black string decays by space pinching off locally at the black string horizon, it will produce a bubble much smaller than the soliton. But since the soliton is BPS in this case, it is a minimum-energy configuration in the space of initial data, so it would be energetically favorable for this bubble to evolve into the soliton. There is still the alternative possibility that there is some other endstate in this case: we have not made any attempt to identify such an alternative endstate explicitly. This seems a particularly interesting issue for further study.

Some final issues concern the stability and uniqueness of the solitons as endstates for the decay. For $m=1$, the soliton is BPS, so it clearly provides a stable endpoint for tachyon condensation for a black string in which the circle along $\xi=\partial_{y}-\frac{1}{R} \partial_{\phi}$ is string scale near the horizon. For larger $m$, the situation is not clear, as the solitons of [17 have not been shown to be stable. However, we might view this

\footnotetext{
${ }^{9}$ The black string is always near-extreme, in the sense that it is close to the point where the horizon area vanishes, but it is far from the BPS bound if $\delta$ is small.
} 
as an indication that they should be stable, so that they can provide an endpoint for the tachyon condensation process. In the case $m=1$, more general smooth solitons which break the rotational symmetry in the $\phi$ and $\psi$ directions have also been constructed [34, 24, 35. It may be possible to reach some of these other BPS solitons as the endpoint of tachyon condensation by turning on the tachyon in a way which breaks the symmetry, although to reach the general soliton solution, this would have to produce a large change in the near-horizon geometry. In the language of the worldsheet RG, there is a set of IR fixed points, and which one we reach depends on the details of the trajectory.

We found no sign that expanding bubble solutions provide an endpoint for tachyon condensation when we have periodic fermions on the asymptotic $S^{1}$. The smooth stationary solitons provide a natural endstate in almost all cases, and in the exceptional case, the soliton is the state of lowest energy. This might have been anticipated, since the positive energy theorem [36] forbids the existence of analogues of the vacuum bubble of [10] in this case. There is a family of vacuum bubble solutions where the circle which shrinks is twisted, constructed by analytic continuation of the Kerr metric [37, 38; however, these solutions always have $|m|<1$, so they do not have a compact circle asymptotically. ${ }^{10}$ However, the standard positive-energy results do not appear to forbid the existence of finite-energy expanding bubbles, so this result is not simply a consequence of those theorems. It is satisfying that we find no sign that considering a localised finite-energy excitation will allow us to initiate such a 'decay to nothing' in the supersymmetric sector. This is similar to results of [39], which found that bubbles constructed from the Euclidean Kerr black hole do not expand into regions with local supersymmetry.

That is, although we find that there are asymptotically supersymmetric solutions with a localised tachyon, the decay of this tachyon does not destroy the spacetime. Rather, the decay process is completely localised (apart from the emission of some excess energy), and leaves the asymptotics of the spacetime unchanged. This is, in fact, an even more localised example of tachyon condensation than the original example of [3].

Acknowledgements: I thank Gary Horowitz for comments and discussion. This work is supported by the EPSRC.

\section{References}

[1] E. J. Martinec, "Defects, decay, and dissipated states," hep-th/0210231.

[2] M. Headrick, S. Minwalla, and T. Takayanagi, "Closed string tachyon condensation: An overview," Class. Quant. Grav. 21 (2004) S1539-S1565, hep-th/0405064.

\footnotetext{
${ }^{10}$ In the Euclidean Kerr black hole, the geometry has periodic identifications along the Killing vector which generates the horizons, $(\tau, \phi) \sim(\tau+2 \pi R, \phi+2 \pi R \Omega)$, where $\Omega$ is the angular velocity of the horizon and $R$ is the inverse of the surface gravity. It is found that $R \Omega<1$ for any choice of parameters, so there is no combination of this identification and $\phi \sim \phi+2 \pi$ which gives an identification simply along $\tau$, which would give a compact circle. See [37, 38] for details.
} 
[3] A. Adams, J. Polchinski, and E. Silverstein, "Don't panic! Closed string tachyons in ALE space-times," JHEP 10 (2001) 029, hep-th/0108075.

[4] J. R. David, M. Gutperle, M. Headrick, and S. Minwalla, "Closed string tachyon condensation on twisted circles," JHEP 02 (2002) 041, hep-th/0111212.

[5] M. Berkooz, Z. Komargodski, D. Reichmann, and V. Shpitalnik, "Flow of geometries and instantons on the null orbifold," hep-th/0507067

[6] A. Adams, X. Liu, J. McGreevy, A. Saltman, and E. Silverstein, "Things fall apart: Topology change from winding tachyons," hep-th/0502021.

[7] G. T. Horowitz, "Tachyon condensation and black strings," hep-th/0506166.

[8] M. S. Costa, C. A. R. Herdeiro, J. Penedones, and N. Sousa, "Hagedorn transition and chronology protection in string theory," hep-th/0504102.

[9] J. McGreevy and E. Silverstein, "The tachyon at the end of the universe," hep-th/0506130.

[10] E. Witten, "Instability of the Kaluza-Klein vacuum," Nucl. Phys. B195 (1982) 481.

[11] A. Saltman and E. Silverstein, "A new handle on de Sitter compactifications," hep-th/0411271.

[12] V. Balasubramanian, J. de Boer, E. Keski-Vakkuri, and S. F. Ross, "Supersymmetric conical defects: Towards a string theoretic description of black hole formation," Phys. Rev. D 64 (2001) 064011, hep-th/0011217.

[13] J. M. Maldacena and L. Maoz, "De-singularization by rotation," JHEP 12 (2002) 055, hep-th/0012025.

[14] O. Lunin, "Adding momentum to D1-D5 system," JHEP 04 (2004) 054, hep-th/0404006.

[15] S. Giusto, S. D. Mathur, and A. Saxena, "Dual geometries for a set of 3-charge microstates," Nucl. Phys. B701 (2004) 357-379, hep-th/0405017.

[16] S. Giusto, S. D. Mathur, and A. Saxena, "3-charge geometries and their CFT duals," Nucl. Phys. B710 (2005) 425-463, hep-th/0406103.

[17] V. Jejjala, O. Madden, S. F. Ross, and G. Titchener, "Non-supersymmetric smooth geometries and D1-D5-P bound states," Phys. Rev. D 71 (2005) 124030, hep-th/0504181.

[18] J. C. Breckenridge, R. C. Myers, A. W. Peet, and C. Vafa, "D-branes and spinning black holes," Phys. Lett. B391 (1997) 93-98, hep-th/9602065. 
[19] J. P. Gauntlett, R. C. Myers, and P. K. Townsend, "Black holes of $D=5$ supergravity," Class. Quant. Grav. 16 (1999) 1-21, hep-th/9810204.

[20] C. A. R. Herdeiro, "Special properties of five dimensional BPS rotating black holes," Nucl. Phys. B582 (2000) 363-392, hep-th/0003063.

[21] M. Cvetic and D. Youm, "Rotating intersecting M-branes," Nucl. Phys. B499 (1997) 253-282, hep-th/9612229.

[22] P. Kraus, F. Larsen, and S. P. Trivedi, "The Coulomb branch of gauge theory from rotating branes," JHEP 03 (1999) 003, hep-th/9811120.

[23] T. Harmark and N. A. Obers, "Thermodynamics of spinning branes and their dual field theories," JHEP 01 (2000) 008, hep-th/9910036

[24] O. Lunin and S. D. Mathur, "AdS/CFT duality and the black hole information paradox," Nucl. Phys. B623 (2002) 342-394, hep-th/0109154.

[25] O. Lunin and S. D. Mathur, "Statistical interpretation of Bekenstein entropy for systems with a stretched horizon," Phys. Rev. Lett. 88 (2002) 211303, hep-th/0202072.

[26] S. D. Mathur, "The fuzzball proposal for black holes: An elementary review," hep-th/0502050.

[27] J. M. Maldacena and H. Ooguri, "Strings in $\mathrm{AdS}_{3}$ and $S L(2, R)$ WZW model. I," J. Math. Phys. 42 (2001) 2929-2960, hep-th/0001053.

[28] J. J. Friess, S. S. Gubser, and I. Mitra, "Counter-examples to the correlated stability conjecture," hep-th/0508220.

[29] M. Cvetic and D. Youm, "General rotating five dimensional black holes of toroidally compactified heterotic string," Nucl. Phys. B476 (1996) 118-132, hep-th/9603100.

[30] M. Cvetic and F. Larsen, "General rotating black holes in string theory: Greybody factors and event horizons," Phys. Rev. D 56 (1997) 4994-5007, hep-th/9705192.

[31] M. Cvetic and F. Larsen, "Near horizon geometry of rotating black holes in five dimensions," Nucl. Phys. B531 (1998) 239-255, hep-th/9805097.

[32] E. J. Martinec and W. McElgin, "String theory on AdS orbifolds," JHEP 04 (2002) 029, hep-th/0106171.

[33] E. J. Martinec and W. McElgin, "Exciting AdS orbifolds," JHEP 10 (2002) 050, hep-th/0206175.

[34] O. Lunin and S. D. Mathur, "Metric of the multiply wound rotating string," Nucl. Phys. B610 (2001) 49-76, hep-th/0105136. 
[35] O. Lunin, J. Maldacena, and L. Maoz, "Gravity solutions for the D1-D5 system with angular momentum," hep-th/0212210.

[36] E. Witten, "A simple proof of the positive energy theorem," Commun. Math. Phys. 80 (1981) 381.

[37] F. Dowker, J. P. Gauntlett, G. W. Gibbons, and G. T. Horowitz, "The decay of magnetic fields in Kaluza-Klein theory," Phys. Rev. D 52 (1995) 6929-6940, hep-th/9507143.

[38] M. S. Costa and M. Gutperle, "The Kaluza-Klein Melvin solution in M-theory," JHEP 03 (2001) 027, hep-th/0012072.

[39] O. Aharony, M. Fabinger, G. T. Horowitz, and E. Silverstein, "Clean time-dependent string backgrounds from bubble baths," JHEP 07 (2002) 007, hep-th/0204158. 